\title{
Compound TCP with Random Losses
}

\author{
Alberto Blanc ${ }^{1}$, Konstantin Avrachenkov², Denis Collange ${ }^{1}$, \\ and Giovanni Neglia ${ }^{2}$ \\ 1 Orange Labs, 905 rue Albert Einstein, 06921 Sophia Antipolis, France \\ \{alberto.blanc, denis. collange\}@orange-ftgroup.com \\ 2 I.N.R.I.A. 2004 route des lucioles, 06902 Sophia Antipolis, France \\ $\{k$.avrachenkov, giovanni.neglia\}@sophia.inria.fr
}

\begin{abstract}
We analyze the performance of a single, long-lived, Compound TCP (CTCP) connection in the presence of random packet losses. CTCP is a new version of TCP implemented in Microsoft Windows to improve the performance on networks with large bandwidth delayproducts. We derive a Markovian model for the CTCP sending window and compute the steady state distribution of the window and the average throughput of a CTCP connection. We observe that the previous approximation, using a "typical cycle," underestimates the average window and its variance while the Markovian model gives more accurate results. We use our model to compare CTCP and TCP Reno. We notice that CTCP gives always a throughput equal or greater than Reno, while relative performance in terms of jitter depends on the specific network scenario: CTCP generates more jitter for moderate-high drop rate values, while the opposite is true for low drop rate values.
\end{abstract}

Keywords: TCP, Compound TCP, Bernoulli Losses, Markov Model.

\section{Introduction}

With the increasing popularity of faster access links like Fiber To The Home [1, the current standard TCP is not always ideal. As indicated by Floyd 2 the current standard is not able to reach high rates in realistic environments, i.e. with typical packet loss rates. Many new transport protocols have been proposed and are currently being studied to replace it. Some of them are already implemented in the latest versions of some operating systems, like Compound TCP (CTCP) on Windows, and Cubic (and others) on Linux. For a survey and comparative analysis of several high speed TCP versions see, for example, 345. For the next few years, the new high speed TCP versions will play an increasing role in resource sharing among flows in the Internet. Yet the behavior, the performance, and the impact on the network of these protocols are not well-known. In particular, there is no comprehensive analytical study of CTCP.

CTCP has been presented by Microsoft Research in [6] and [7] in 2006. It is currently submitted as a draft to the IETF Network Working group with minor differences [8]. CTCP is enabled by default in computers running Windows Server 2008 and disabled by default in computers running Windows Vista 9]. It 
is also possible to add support for CTCP to Windows XP. An implementation of CTCP, based on 78, is also available for Linux 10. The main objective of the authors of CTCP [7] is to specify a transport protocol which is efficient, using all the available bandwidth, fair and conservative, limiting its impact on the network. They propose to combine the fairness of a delay-based approach with the aggressiveness of a loss-based approach. As the proposal of CTCP is still recent, there are only a few published evaluations of it. The only analytical model of CTCP in [7] is based on a de facto deterministic model.

In the present work we study the performance of CTCP under random losses. This model has been widely used in the literature (e.g. [111213]) to analyze the influence of random traffic fluctuations on the performance of TCP Reno. While it is not necessarily the most sophisticated model, it does capture the behavior of TCP Reno in several real cases (see, for example, [11]3]). Whether the same holds true for more recent versions of TCP is an open question, to the best of our knowledge. The present work is just a first attempt at studying the influence of random losses on long-lived CTCP connections. These losses can be caused by a large number of connections sharing the same bottleneck link or by transmission errors in wireless networks. As new physical layer rates keep increasing thanks to new technologies like WiMax, wireless networks can have larger bandwidth delay products, reaching values for which the behavior of new versions of TCP differs from Reno. (In the case of CTCP if the window is less than 41 packets CTCP behaves like Reno.)

The outline of the paper and of our results is as follows. In Section 2 we give a brief overview CTCP. In Section 3 we present a two-dimensional Markov chain model for CTCP. With the help of this model we obtain the long-run average throughput of CTCP and the distribution of its congestion window at congestion events. Then, in Section 3.2 we use Palm calculus to obtain the distribution of the congestion window at arbitrary time moments. In Section 3.3 we propose some heuristics and compare them with the accurate two-dimension Markov chain model. Finally, in Section 4 we provide numerical and simulation results which confirm our theoretical findings. In particular, we conclude that CTCP provides a higher throughput than TCP Reno and, even if more aggressive, it causes less traffic jitter than TCP Reno on high speed links with small random losses.

Due to space constraints, some technical details are provided in a companion technical report [14].

\section{Compound TCP Overview}

In this section we give a very brief overview of CTCP (see [7] for a complete description). The main idea of CTCP is to quickly increase the window as long as the network path is not fully utilized, then to keep it constant for a certain period of time and finally to increase it by one MSS (Maximum Segment Size) per round trip time just like TCP Reno. We will often use the term "phases" for these three different behaviors. 
During phase 1 the sender computes the sending window $(w)$ at the $(i+1)$-th round trip as $w_{i+1}=w_{i}+\alpha w_{i}^{k}$ (as suggested in [7] we use $\alpha=1 / 8$ and $k=3 / 4$ ). At each round trip the sender estimates the bandwidth-delay product and the amount of data backlogged in the network using the same method adopted by TCP Vegas [15. If the amount of backlogged data is greater than a certain threshold $(\gamma$, usually set to 30 [7 16]) the sender switches to phase 2 and keeps the window constant. This constant value corresponds to the sum of the estimated bandwidth-delay product and the estimated amount of backlogged data. We consider an ideal behavior of CTCP, assuming that such estimates are correct. In such case the window in phase 2 is equal to $\theta \triangleq \mu \tilde{\tau}+\gamma$, where $\mu$ is the capacity of the bottleneck link and $\tilde{\tau}$ is the round trip propagation delay. In reality any queue size estimate available at the sender is outdated due to feedback delays, this fact combined with the CTCP algorithm presented in [7] causes the window to oscillate during this phase as we analyzed in [17. The length of phase 2 is dictated by the "congestion component" of the window. In fact in CTCP the congestion window $w$ is the sum of two components: the delay window $w_{d}$ and the congestion window $w_{c}$. The congestion component is incremented by one every round trip (just like the TCP Reno congestion window) and when this component reaches $\theta$ phase 2 ends. The delay component is set such that the value of the total window $\left(w_{i}=w_{c_{i}}+w_{d_{i}}\right)$ at the $i$-th round trip time follows the following evolution in absence of packet losses:

$$
w_{i}= \begin{cases}w_{i-1}+\delta_{i} & , \text { if } w_{i-1}+\delta_{i}<\theta \\ \theta & , \text { if } w_{i-1}+\delta_{i} \geq \theta \text { and } w_{c_{0}}+i<\theta \\ w_{i-1}+1 & , \text { otherwise }\end{cases}
$$

where $\delta_{i}=\max \left\{\left\lfloor\alpha w_{i-1}^{k}\right\rfloor, 1\right\}$. Figure 1 shows the three phases of the window evolution. When a loss occurs both the total window and the congestion window are halved.

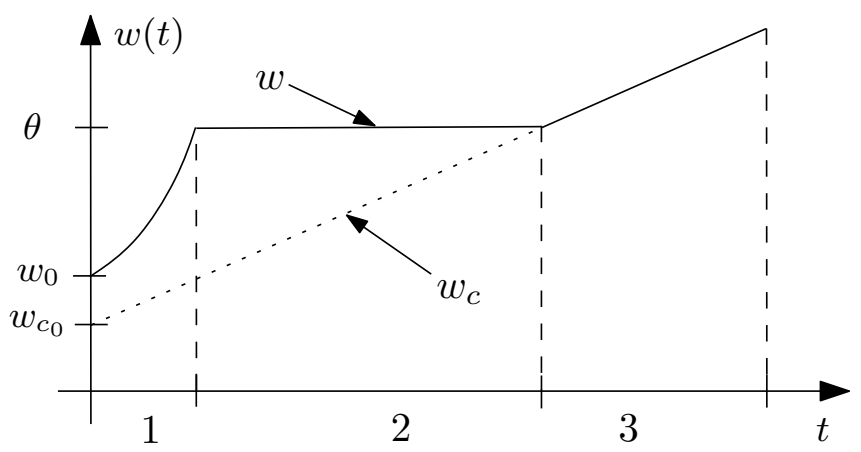

Fig. 1. The evolution of $w$ and $w_{c}$ in CTCP 


\section{Performances with Random Losses}

We consider a single long lived TCP compound flow using a path with $\mu \tilde{\tau}$ bandwidth delay product and buffer size equal to $b$. The flow will experience a loss every time that its window size reaches the value $\mu \tilde{\tau}+b$. For this reason, we can consider $w_{\max }=\mu \tilde{\tau}+b$ as an upper bound for the the window size. Beside the deterministic losses due to buffer overflow, we consider also that each packet can be dropped with some probability $p$, independently from all other packets, i.e. according to a Bernoulli process. In what follows we derive the throughput and the window distribution in steady state. We are going to assume that $w$ can only take integer values.

\subsection{Throughput Calculation}

We define a cycle as the time interval between two consecutive losses. We denote as $w_{c_{n}}^{t}$ (respectively $w_{d_{n}}^{t}$ ) the congestion (respectively delay) window at the begin of the $(n+1)$-th round trip time of the $t$-th cycle. We will omit the superscript $t$ whenever it is clear which cycle is being considered.

We observe that in our framework the evolution of the window in each cycle $t$ depends from previous cycles only through the window value at the begin of the cycle, or, more precisely, through the two initial values $w_{c_{0}}^{t}$ and $w_{d_{0}}^{t}$, which can be determined by the final value of the windows at the $t-1$-th cycle. This also implies that it is possible to use the renewal reward theorem to compute the average throughput as (see [13]):

$$
E[\lambda]=\frac{E[S]}{E[T]}
$$

where $\lambda$ is the throughput (in MSS/s), $S$ is the total number of packets sent during a cycle and $T$ is the duration of the cycle.

Both $E[T]$ and $E[S]$ can be evaluated starting from the knowledge of the distribution size of the two (correlated) random variables $W_{c_{0}}$ and $W_{d_{0}}$. We denote $g\left(w_{c_{0}}, w_{d_{0}}\right)$, the probability mass function ( $\left.\mathrm{pmf}\right)$ of these random variables. We first show how to derive the distribution of cycle duration from $g\left(w_{c_{0}}, w_{d_{0}}\right)$ and then derive the pmf $g()$ itself. $E[S]$ can be evaluated similarly to $E[T]$.

A cycle has length equal to $n$ if there is a loss at the $n$-th round trip time. As we are assuming that there is a loss whenever $w=w_{\max }$, all cycles have a finite length. Let $m\left(w_{0}\right)=\min \left\{n \mid w_{n} \geq w_{\max }\right\}$ be the maximum possible length (in round trips) of a cycle starting at $w_{0}$. For $n<m\left(w_{0}\right)$ the probability of a cycle having length equal to $n$ can be derived from the Bernoulli loss process as:

$$
\begin{aligned}
P\left[T=n \mid W_{c_{0}}=w_{c_{0}}, W_{d_{0}}=w_{d_{0}}\right] & =(1-p)^{V_{n-1}\left(w_{0}\right)}-(1-p)^{V_{n}\left(w_{0}\right)} \\
& \triangleq a_{n}\left(w_{c_{0}}, w_{d_{0}}\right),
\end{aligned}
$$

where

$$
\mathrm{V}_{n}\left(w_{0}\right) \triangleq \sum_{i=0}^{n-1} w_{i}, V_{0} \triangleq 0
$$


and $w_{i}$ is computed as in (11) so that $V_{n}$ is the number of packets sent during the $n$-th round trip of a cycle starting with $w=w_{0}$. Both $V_{n}$ and $a_{n}$, as most quantities used in this section, depend on the initial window $w_{0}=w_{c_{0}}+w_{d_{0}}$ as highlighted by the notation $a_{n}\left(w_{0}\right)$ and $V_{n}\left(w_{0}\right)$, even though we will also use the simplified notation $a_{n}$ and $V_{n}$.

The probability that a loss occurs at the $m\left(w_{0}\right)$-th round trip can be evaluated simply considering that $\sum_{i=1}^{m} P[T=i]=1$ :

$$
\left.P\left[T=m_{(} w_{0}\right) \mid W_{c_{0}}=w_{c_{0}}, W_{d_{0}}=w_{d_{0}}\right]=1-\sum_{i=1}^{m_{\left(w_{0}\right)-1}} a_{i}\left(w_{c_{0}}, w_{d_{0}}\right) .
$$

Finally, being that the support of the discrete random variables $W_{c_{0}}$ and $W_{d_{0}}$ is finite, we can use a finite sum to compute $P[T]$ :

$$
P[T=n]=\sum_{w_{c_{0}}, w_{d_{0}}} a_{n}\left(w_{c_{0}}, w_{d_{0}}\right) g\left(w_{c_{0}}, w_{d_{0}}\right) .
$$

In order to compute $g\left(w_{c_{0}}, w_{d_{0}}\right)$ we model the evolution of the window (at the beginning of each cycle) with a Markov chain. The evolution of the window of TCP Reno at the begin of a cycle $\left(w_{0}^{t}\right)$ has been modeled in other works as a Markov chain (see, for example, [1213]). In fact $w_{0}^{t}$ is equal to half of the window value at the end of the $(t-1)$-th cycle, which depends only on $w_{0}^{t-1}$ and on packet loss probability $p$.

In order to model CTCP we use a two-dimensional discrete Markov chain $X_{t}$ to account for $w_{c_{0}}^{t}$ and $w_{d_{0}}^{t}$. For each state $(i, j)$ the first index represents $w_{c_{0}}^{t}$ and the second $w_{d_{0}}^{t}$. For any pair of states it is possible to compute the transition probability as:

$$
P\left[X_{t+1}=(k, l) \mid X_{t}=(i, j)\right]=\sum_{n \in B} a_{n}(i, j)
$$

with $\left.w_{c_{0}}^{t}=i, w_{d_{0}}^{t}=j, B=\{n\rfloor\left\lfloor w_{c_{n}}^{t} / 2\right\rfloor=k,\left\lfloor w_{d_{n}}^{t} / 2\right\rfloor=l\right\}$, where $w_{c_{n}}^{t}$ and $w_{d_{n}}^{t}$ are evaluated according to (11). The sum on the right hand side is needed because different pairs $\left(w_{c_{n}}^{t}, w_{d_{n}}^{t}\right)$ can originate, after a loss, the same pair $\left(w_{d_{0}}^{t+1}, w_{d_{0}}^{t+1}\right)$ as we use integer values for the window. As $w \leq w_{\max }$ we have that $w_{c_{0}} \leq$ $\left\lfloor w_{\max } / 2\right\rfloor \triangleq N$ and, if $\theta$ is the value of the window during the constant window phase, $w_{d_{0}} \leq\lfloor\theta / 2\rfloor \triangleq M\left(\right.$ as $\left.w_{d} \leq \theta\right)$. Combining these two bounds we obtain that the number of states in the Markov chain is $N M$. Using the ARPACK implementation of the Arnoldi method [18] it is possible to efficiently calculate the steady state distribution of the Markov chain $X_{t}$ even for large values of $N M$. The more time consuming step is actually to compute the transition matrix for $X$. The complexity of the algorithm we used is $O\left(M N^{2}\right)$; we believe that it is not possible to decrease the complexity of the algorithm given that it has to compute all the possible transitions and these grow like $M N^{2}$. Note that the number of possible transitions for a Markov chain with $N M$ states is $N^{2} M^{2}$ therefore we already take into account that, in this case, some transitions are not possible.

Once the steady state distribution of the Markov chain $X_{t}\left(g\left(w_{c_{0}}, w_{d_{0}}\right)\right)$ is derived, we can use it to compute $E[T], E[S]$ and then the average throughput using (2). 
We observe that so far we have implicitly measured $T$ in terms of number of round trip times, but we can slightly change our model in order to consider real time duration (seconds) taking into account also queuing delay variation due to the TCP flow itself [14.

\subsection{Steady State Distribution of the Window}

In the previous section we have described how to compute the steady state distribution of $w_{c_{0}}$ and $w_{d_{0}}$, and consequently also the value of the window $w_{0}=w_{c_{0}}+w_{d}$ at the beginning of each cycle. In this section we are interested in the steady state distribution of the window as a function of time. We denote as $Y_{n}$ the value of the window at the begin of the $(n-1)$-th round trip time. Note that $Y_{n}$ is different both from $X_{t}$, which is the value of the window after a packet loss, and from $w_{n}^{t}$, which is the value of the window at the begin of the $(n-1)$-th round trip time in the $t$-th cycle. Clearly $X_{t}$ represents a subsequence of the sequence $Y_{n}$. We observe that $Y_{n}$ can also be modeled as a discrete time Markov chain where a transition occurs every round trip. Also this Markov chain is ergodic, hence it admits a steady state and we assume that it is in steady state at time 0 .

Using Palm calculus, we first compute $P\left[Y_{n}=k\right]$ starting from $P\left[W_{0}=w_{0}\right]$, where $Y_{n}$ represents the window after $n$ round trip times starting from some arbitrary value (given that all the Markov chains involved are ergodic, the initial value is irrelevant).

Let $Z_{n}$ be the (discrete) time of the $n$-th packet drop after time 0 . Using the intensity and inversion formulas of Palm calculus [19] we can compute $P\left[Y_{n}=k\right]$ as a function of $P\left[W_{0}=w_{0}\right]$ :

$$
\begin{aligned}
P\left[Y_{n}=k\right] & =E\left[1_{\left\{Y_{n}=k\right\}}\right]=P\left[Z_{0}=0\right] E^{0}\left[\sum_{s=1}^{Z_{1}} 1_{\left\{Y_{s}=k\right\}}\right] \\
& =\eta E^{0}\left[\sum_{s=1}^{Z_{1}} 1_{\left\{Y_{s}=k\right\}}\right] \\
& =\eta \sum_{w_{0}, l}\left[P\left[W_{0}=w_{0}\right] P\left[Z_{1}=l \mid W_{0}=w_{0}\right] \sum_{s=1}^{l} 1_{\left\{Y_{s}=k \mid W_{0}=w_{0}\right\}}\right]
\end{aligned}
$$

where $E^{0}$ is the Palm expectation, $1_{\left\{Y_{n}=k\right\}}$ is the indicator function for the event $Y_{n}=k$ and $\eta$ is the intensity of the process $Z_{n}$. The second (5) and third (6) equalities follow from the inversion and intensity formulas, respectively, while (7) follows from the total probability theorem, conditioning on all the possible values of $W_{0}$ and $l$. Given that $Z_{n}$ is an ergodic process $P\left[Z_{0}=0\right]$ can be computed, using the intensity formula, as the inverse of the expected value of $T \stackrel{\mathrm{d}}{=} Z_{n}-Z_{n-1}$ that is as the average length of a cycle (in round trips) so that $\eta=1 / E[T]$ where $E[T]$ can be computed using (4).

As for the calculations in section 3.1 we can evaluate the distribution of the window taking into account the effect of queueing delays as well [14]. 


\subsection{A Simple Approximation and the Deterministic Response Function}

The method described in the previous section provides an exact solution, but it can be computationally expensive for medium and large values of $w_{\max }$ and $\theta$. Using the same method as in [12] it is possible to quickly find an approximate solution for the average window size. The idea is to consider a sequence of "typical" or "average" cycle. If $p$ is the probability that a packet is dropped, the average cycle has exactly $1 / p$ packets (provided the probability of reaching $w_{\max }$ is negligible). Let us denote $w_{0}$ the initial window of an average cycle and $w_{n}\left(w_{0}\right)$ the final window, after $n$ round trip times during which $1 / p$ packets are transmitted. Being that the following average cycle has to be identical and that CTCP, as Reno, halves the window at the end of each cycle, it has to be:

$$
w_{n}\left(w_{0}\right)=2 w_{0} .
$$

Imposing the constraint $V_{n}\left(w_{0}\right)=1 / p\left(V_{n}\left(w_{0}\right)\right.$ is defined in section 3.1), we can identify the unique possible value of $w_{0}$ and then the unique possible window evolution corresponding to $p$. Once the window evolution is known, the average window can be obtained and can be plotted as a function of the drop rate.

As previously noted in [11] this approach corresponds to the calculation of what is usually called the "deterministic response function." This function is often used in the literature on TCP. For example, 2] defines the response function as "the function mapping the steady-state packet drop rate to TCP's average sending rate in packets per round-trip time." Where the drop rate is simply the inverse of the number of packets sent during each cycle. In this case the term "drop rate" always refers to this quantity and not to any probabilistic model, while this usage is not the most appropriate one it is, nonetheless, common in the literature. From another point of view, we observe that the average cycle corresponds to the actual evolution of the window under our loss model, when there is no random loss, but the bandwidth delay product and the buffer size are such to cause a deterministic loss every $1 / p$ packets.

When the window update rule operates on a round trip time basis - as in CTCP but also in Reno and HighSpeed for example - the deterministic response function does not depend on physical parameters like capacity or propagation delay. In other words it can be considered as an intrinsic property of the specific growth function used to increment the window. On the contrary, for TCP Cubic the growth of the window depends on real time and hence also on link capacities and propagation delays, so that a comparison with the TCP versions indicated above would need special care.

Figure 2 shows the response function for different values of $\theta$, between 100 MSS and 1000 MSS (Maximum Segment Size). The two lines correspond to two different ways to express the window evolution in (8). The dotted line corresponds to a fluid model where the growth of the window is approximated with a continuous function (see 20]). More precisely

$$
w_{n}\left(w_{0}\right)=\left((1-k) \alpha n+w_{0}^{* 1-k}\right)^{\frac{1}{1-k}} .
$$




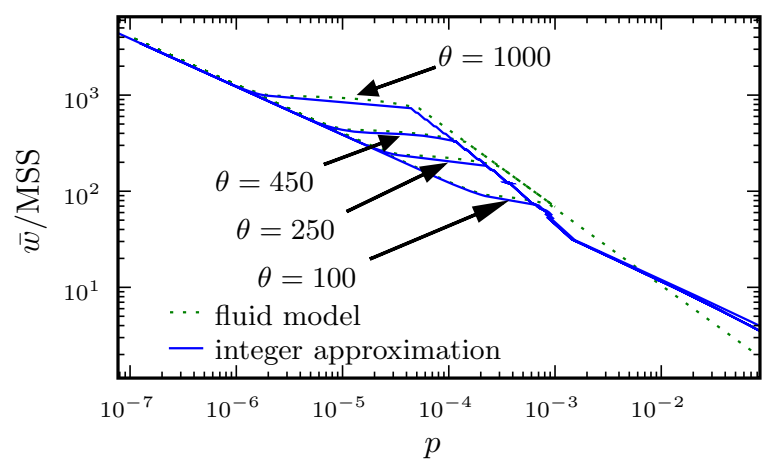

Fig. 2. The deterministic response function for $\theta=100,250,450,1000$

The solid line, instead, considers only integer values of the window and computes the increment of the window as according to (1) with $w_{c_{0}}=w_{0}$. In this case the last round trip time of the cycle -the $n$-th one- is evaluated as $n=\min \{i$ : $\left.w_{i} \geq 2 w_{0}\right\}$. In both cases we have considered $w_{0}$ as the independent value. For each value of $w_{0}$ we have then computed $S$, the total amount of packets sent during a cycle starting with $w=w_{0}$ and then we have plotted the average window as a function of $1 / S=p$. Clearly the total number of packets sent during such a cycle is a monotonically increasing function of $w_{0}$ (as the TCP window is monotonically increasing during each cycle) so that increasing values of $w_{0}$ correspond to increasing values of $S$, and decreasing values of $p=1 / S$.

Regarding the integer approximation we observe that, for $\alpha=1 / 8$ and $k=3 / 4$ (values suggested in [7]), $\alpha w^{k} \geq 2$ only if $w>30$. That is the CTCP window grows by one each round trip, the same as Reno, as long as $w<30$. This is somewhat consistent with what suggested in [7] where the authors call for the delay component to be used (that is to increment the window by $\alpha w^{k}$ ) only if the window is larger than lowwnd which they set equal to 41 . This observation explains why for small values of $w$ the fluid and integer approximation are different, with the integer approximation giving a larger average value of the window.

In the case of the integer approximation and for large drop rates (more than $10^{-3}$ ) the response function is the same as Reno. The same is true, regardless of the approximation, for small drop rates (less than $10^{-6}$ ) given that, in this latter case, the evolution of the window is the same in CTCP and Reno. For drop rates roughly between $4 \cdot 10^{-4}$ and $10^{-3}$ only the rapid increase phase of CTCP is used so that the response function is steeper. For drop rates between $10^{-6}$ and $4 \cdot 10^{-4}$ the "constant" window phase is used along with all the other phases and this explains why the response function increases more slowly until it reaches the Reno response function.

While for Reno and HighSpeed TCP the response function is only a function of the drop rate, for CTCP the response function is also a function of $\theta$ (the value of the window during the constant window phase). The larger the value of $\theta$ the longer the rapid increase phase can be. In the extreme case of $\theta=\infty$ the other 
phases would not take place at all and the response function would be much steeper. Note that in [7] the authors compute the response function for exactly this case $(\theta=\infty)$. Given that they are interested in limiting the aggressiveness of CTCP, they are in effect considering the worst case, by only considering the rapid increase phase. At the same time it can be argued that, as a consequence, CTCP is less aggressive than HighSpeed TCP as, for small values of $p$, CTCP is as aggressive Reno, which is less aggressive than HighSpeed TCP.

Finally it is worth noting that, as $p$ decreases, the difference between the fluid and integer models for the rapid increase phase becomes negligible.

\section{Numerical Results}

Using the models presented in the previous sections we can compute the average throughput and the average window size for different values of the drop probability $p$. Figure 3 shows the CTCP response function computed using the two deterministic models presented in section 3.3 and the probabilistic model discussed in section 3.1. for $\theta=250 \mathrm{MSS}$. For the same "drop probability" the deterministic model with integer approximations gives a smaller average window than the probabilistic model, which uses the very same integer approximations, as already observed in [11. The fluid model, instead, does agree with the probabilistic model, for larger values of $p$. As discussed in the previous section, the fluid model overestimates the window growth for certain values of $p$ (roughly between $10^{-} 4$ and $10^{-} 2$ ). We cannot explain why this overestimation is almost in agreement with the probabilistic model and we believe that it is just a coincidence.

The probabilistic and deterministic models do have almost the same values for values of $p$ around $2 \cdot 10^{-} 4$. This can be explained by the fact that, for these values of $p$, most of the drops take place during the "constant window" phase. In this case even if the cycles have a different length the average window size is the same: random drops do change the cycle length but not the average window size.

One should take some care in comparing these two models: in the case of the deterministic model the buffer size at the bottleneck is fixed (so that all the

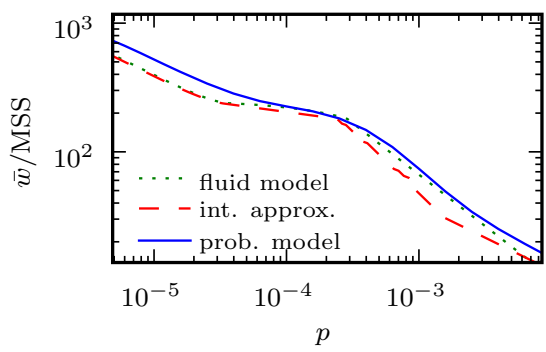

Fig. 3. CTCP response function

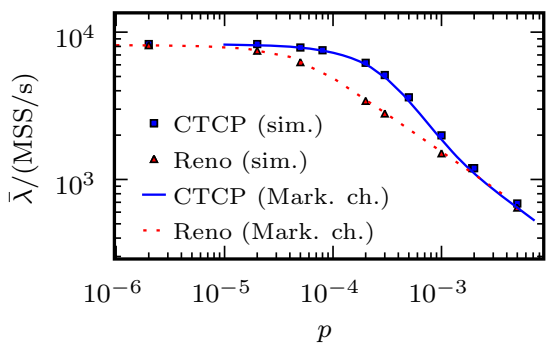

Fig. 4. Average Throughput 
cycles have the same size) while in the case of the probabilistic model the buffer size at the bottleneck link is much larger (in theory infinite, set to $1600 \mathrm{MSS}$ for the numerical results) and allows the window to reach larger values. If we used the same buffer size in both models the average window would be smaller in the probabilistic case.

Figure 4 shows the average throughput for CTCP and TCP Reno. For CTCP we have used the probabilistic model introduced in section 3.1 while for Reno we have used an equivalent model but with a "one dimensional" Markov chain as Reno does not have two components in the congestion window. The squares and triangles in Figure 4 correspond to simulation results. For both versions of TCP there is a good match between the probabilistic model and the simulations, obtained using ns-2 (version 2.33) with a Linux implementation of CTCP [10]. The simulations use the classical "dumbbell" topology with a single source and a single destination. There is a single TCP connection with no cross traffic going through a bottleneck of $100 \mathrm{Mb} / \mathrm{s}$ with propagation delay of $26.4 \mathrm{~ms}$ and where each packet of $1500 \mathrm{~B}$ is dropped with probability $p$. The duration of each simulation is $200000 \mathrm{~s}$. As the difference between different simulation runs is very small (less than 1\%) we did not plot errorbars. As the simulations and the model share the same assumptions they can only be used as a sanity check. While assessing the influence of the assumptions used in the model and how realistic they are is a very interesting problem it is outside the scope of this work.

In Figure $4 w_{\max }=370 \mathrm{MSS}$, the bandwidth-delay-product is $220 \mathrm{MSS}$ and the buffer size is $150 \mathrm{MSS}$, while in Figure $3 w_{\max }=1600 \mathrm{MSS}$. This explains why in Figure 4 the throughput is constant for small drop probabilities (most of the packets are dropped when $w=w_{\max }$ ) a similar behavior takes place for larger values of $w_{\max }$ but for drop probabilities smaller than those included in Figure 3 .

Figures [5] and 6] show the distributions for $w_{0}, w_{c_{0}}$ and $w_{d_{0}}$ when $\theta=430 \mathrm{MSS}$ and $w_{\max }=600 \mathrm{MSS}$ for $p=3 \cdot 10^{-4}$ and $p=5 \cdot 10^{-6}$ respectively. The dotted lines represent simulation results, confirming that there is a good match with the Markov model. Figure 5 corresponds to the case where most of the packet are dropped during phase 1 when the window is growing quickly. In this case the

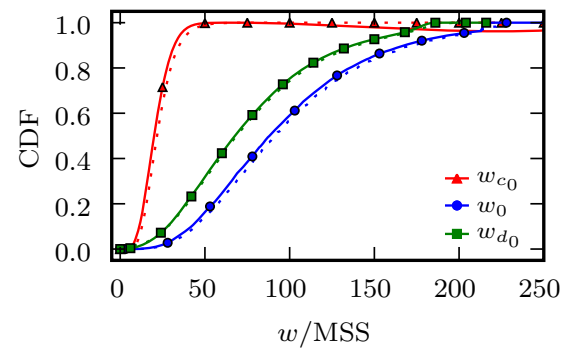

Fig. 5. Distribution of $w_{0}, w_{c_{0}}$ and $w_{d_{0}}$ $\left(\theta=430 \mathrm{MSS}, w_{\max }=600 \mathrm{MSS}, p=\right.$ $\left.3 \cdot 10^{-4}\right)$

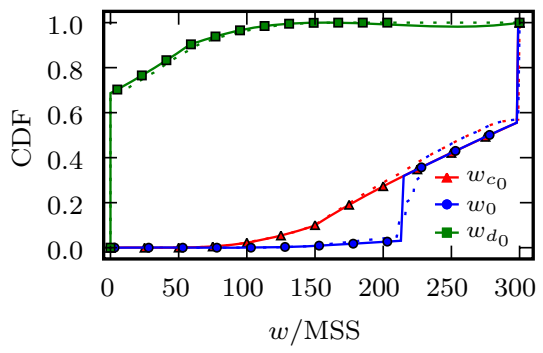

Fig. 6. Distribution of $w_{0}, w_{c_{0}}$ and $w_{d_{0}}$ $\left(\theta=430 \mathrm{MSS}, w_{\max }=600 \mathrm{MSS}, p=\right.$ $\left.5 \cdot 10^{-6}\right)$ 


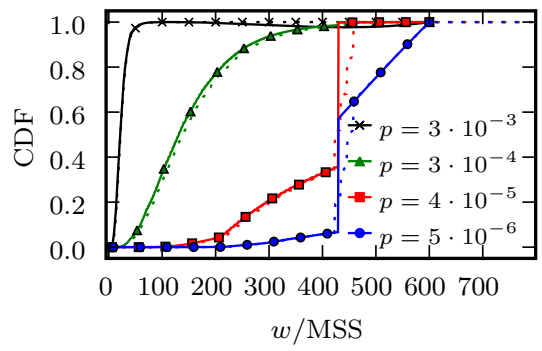

Fig. 7. Distribution of $Y_{n}(\theta=430 \mathrm{MSS}$, $\left.w_{\max }=600 \mathrm{MSS}\right)$

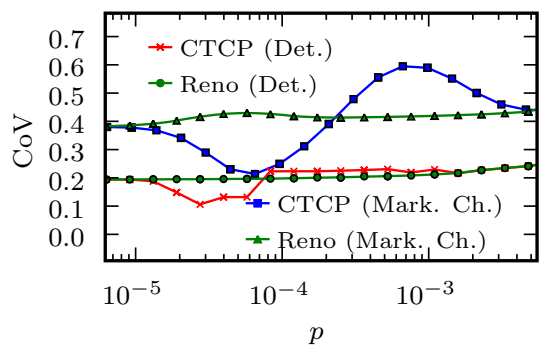

Fig. 8. Coefficient of variation of $Y_{n}$ for CTCP and Reno

distribution of $w_{c_{0}}$ is greater than the distribution of $w_{d_{0}}$, so that, on average, $w_{c_{0}}<w_{d_{0}}$. Figure [6] corresponds to the case where a significant fraction of packets is dropped during the Reno phase (almost 50\%). The jump at $w_{0}=$ 215 MSS represents the packets dropped during the constant window phase (with the simulations having smaller jumps caused by the oscillations of the window). The jump at $w=300 \mathrm{MSS}$ correspond to the case when packets are dropped due to a buffer overflow (recall that in this case $w_{\max }=600 \mathrm{MSS}$ and for the simulations the buffer size $b$ is $200 \mathrm{MSS}$ and $\mu \tilde{\tau}=400 \mathrm{MSS})$. In this case the distribution of $w_{c_{0}}$ is smaller than the distribution of $w_{d_{0}}$, the opposite of what happens in the previous case.

Figure 7 shows the steady state distribution of $Y_{n}$ for different values of $p$ with $\theta=430 \mathrm{MSS}$ and $w_{\max }=2000 \mathrm{MSS}$. Again the dotted lines represent the same distribution for the corresponding ns- 2 simulations. As expected, with increasing drop probabilities, each distribution is strictly greater than all the previous ones. In Figures [6 and 7 while the probabilistic models have a sharp jump for $w=215 \mathrm{MSS}$ and $w=430 \mathrm{MSS}$ the simulations (dotted lines) have smaller jumps. This is caused the oscillations of the sending window during phase 2 in the simulations. As mentioned in section 2 this is consistent with the CTCP algorithm but it is not taken into account by the probabilistic model. While it is, at least in principle, possible to incorporate this aspect into the model, we prefer using a simpler model with a constant value during phase 2 given that the differences between this simplified model and the simulations are not significant (especially as far as the throughput is concerned).

Figure 8 show the coefficient of variations $(\mathrm{CoV})$ for CTCP $(\theta=250 \mathrm{MSS}$, $\left.w_{\max }=2000 \mathrm{MSS}\right)$ and Reno for $\mu \tilde{\tau}=220 \mathrm{MSS}$. In this cases the difference between the deterministic and probabilistic model is more pronounced than in the case of the average window (response function). This can be explained by the fact that the average window depends only the first moment of $Y_{n}$ while the $\mathrm{CoV}$ depends on the second moment as well. For the $\mathrm{CoV}$, in particular, the difference between the two models is significant. For small values of $p$ the CoV of CTCP is smaller than Reno but for larger values of $p$ the opposite is true indicating that for $p>10^{-4} \mathrm{CTCP}$ might not be the best solution. 


\section{Conclusions and Future Work}

In this paper we have presented a Markovian model of CTCP under random losses. This kind of model is a first attempt to roughly assess the impact of varying network conditions on a CTCP connection. The network is seen as a black box randomly dropping packets, due to buffer overflows. This model could also be used to describe the impact of transmission errors in some "challenging environments" (e.g. wireless networks) as expected from new TCP versions 21.

In this first analysis, we have assumed that the loss arrivals follow a simple Bernoulli process. We have computed the distribution of the sending window on loss events with a Markovian model, and then the average throughput. Using Palm Calculus we have computed the steady state distribution of the window. Its value has a direct influence on the buffer occupancy and on the jitter experienced by all the flows sharing the same bottleneck link.

This analysis can be extended in many ways. The Bernoulli loss process could be replaced with a more bursty and realistic process. In this case, multiple losses could take place during the same round-trip time, and the recovery time could be longer. We could also consider time-outs in the case of high loss rates, as in [13. A similar analytical study could also be applied to the other TCP versions, currently under standardization, comparing their efficiency and their robustness.

\section{References}

1. FFTH: Fiber to the home council (2008), http://www.ftthcouncil.org/

2. Floyd, S.: HighSpeed TCP for Large Congestion Windows. RFC 3649 (2003) (Experimental)

3. Kherani, A., Prabhu, B., Avrachenkov, K., Altman, E.: Comparative study of different adaptive window protocols. Telecomm. Systems 30, 321-350 (2005)

4. Li, Y., Leith, D., Even, B.: Evaluating the performance of TCP stacks for highspeed networks. In: Proc. 4th Int. Workshop on Protocols for FAST Long-Distance Networks (2006)

5. Li, Y., Leith, D., Shorten, R.: Experimental evaluation of tcp protocols for highspeed networks. IEEE/ACM Trans. Netw. 15, 1109-1122 (2007)

6. Tan, K., Song, J., Zhang, Q., Sridharan, M.: Compound TCP: A scalable and TCPfriendly congestion control for high-speed networks. In: Proc. 4th Int. Workshop on Protocols for FAST Long-Distance Networks (2006)

7. Tan, K., Song, J., Zhang, Q., Sridharan, M.: A compound tcp approach for highspeed and long distance networks. In: INFOCOM (2006)

8. Sridharan, M., Tan, K., Bansal, D., Thaler, D.: Compound TCP: A new TCP congestion control for high-speed and long distance networks. Internet draft, Internet Engineering Task Force (2007) (work in progress)

9. Davies, J.: Performance enhancements in the next generation TCP/IP stack. The Cable Guy (2007), http://www.microsoft.com/technet/community/columns/ cableguy/cg1105.mspx

10. Andrew, L.: Compound TCP Linux module (2008), http://netlab.caltech.edu/lachlan/ctcp/

11. Altman, E., Avrachenkov, K., Barakat, C.: A stochastic model of tcp/ip with stationary random losses. IEEE/ACM Trans. Netw. 13(2), 356-369 (2005) 
12. Lakshman, T., Madhow, U.: The performance of tcp/ip for networks with high bandwidth-delay products and random loss. IEEE/ACM Transactions on Networking 5, 336-350 (1997)

13. Padhye, J., Firoiu, V., Towsley, D., Kurose, J.: Modeling tcp reno performance: a simple model and its empirical validation. IEEE/ACM Transactions on Networking 8, 133-145 (2000)

14. Blanc, A., Avrachenkov, K., Collange, D., Neglia, G.: Compound tcp with random losses. INRIA Research Report 6736, INRIA (2008), http://hal.inria.fr/docs/00/34/98/45/PDF/RR-6778.pdf

15. Brakmo, L.S., Peterson, L.L.: Tcp vegas: end to end congestion avoidance on a global internet. IEEE JSAC 13, 1465-1480 (1995)

16. Tan, K., Song, J., Sridharan, M., Ho, C.: CTCP-TUBE: Improving TCPfriendliness over low-buffered network links. In: Proc. 6th Int. Workshop on Protocols for FAST Long-Distance Networks (2008)

17. Blanc, A., Collange, D., Avrachenkov, K.: Oscillations of the sending window in Compound TCP. In: Proc. 2nd NetCoop Workshop (2008)

18. Sorensen, D., Lehoucq, R., Yang, C., Maschhoff, K.: ARPACK (2008), http://www. caam.rice.edu/software/ARPACK/

19. Boudec, J.Y.L.: Understanding the simulation of mobility models with palm calculus. Perform. Eval. 64, 126-147 (2007)

20. Blanc, A., Collange, D., Avrachenkov, K.: Modelling an isolated Compound TCP connection. Tech. Report 6736, INRIA (2008), http://hal.inria.fr/docs/00/35/00/41/PDF/RR6736.pdf

21. Floyd, S., Allman, M.: Specifying New Congestion Control Algorithms. RFC 5033 (Best Current Practice) (2007) 